\title{
Effects of Ginger Fortification of Ogi on Lactic Acid Bacteria and Aflatoxin Levels
}

\author{
Oluwafemi F*, Ademu C and Omeike SO \\ Microbiology department, Federal University of Agriculture, PMB 2240, Abeokuta, \\ Ogun State, Nigeria
}

*Corresponding author: Oluwafemi F, Microbiology department, Federal University of Agriculture, PMB 2240, Abeokuta, Ogun State, Nigeria, Tel: +2348035028602; Email: foluwafemi2000@yahoo.co.uk

\section{Abstract}

The influence of ginger on Lactic Acid Bacteria (LAB) load / some probiotic properties, aflatoxin levels and acceptability of ogi (maize pap) co - fermented with ginger was studied. Various quantities $(0,4,6,8$, and 10 grams) of fresh ginger were incorporated into 400 grams fermenting maize at the beginning of fermentation. Changes in Total Titratable Acidity (TTA), pH and LAB load were determined using standard procedures. Sensory evaluation was done using nine point hedonic scales. Enzyme Linked Immunosorbent Assay (ELISA) kit was used to determine the total aflatoxin level. Isolates were screened for probiotic properties and identified using standard procedures. The $\mathrm{pH}$ values ranged between 4.90 and 7.20 in non ginger fermenting maize (control) and from $3.80-7.10$ in the ginger fermenting maize. TTA ranged between $0.11 \%$ and $0.70 \%$ in the control and from $0.12-0.72 \%$ in the ginger fermenting maize. LAB population increase significantly at $\mathrm{p}<0.05$ and ranged from $3.0 \times 104 \mathrm{cfu} / \mathrm{mL}-8.2 .0 \times 104 \mathrm{cfu} / \mathrm{mL}$ in the control and from $3.3 \times$ $104 \mathrm{cfu} / \mathrm{mL}-8.8 \times 104 \mathrm{cfu} / \mathrm{mL}$ in the ginger samples. LAB isolates were identified as Lactobacillus fermentum, $\mathrm{L}$. plantarum, L. acidophilus and L. brevis and they possessed potentials to assimilate cholesterol except L. brevis. They were also able to withstand $\mathrm{pH}$ values of $2,3,4$, and 7 and tolerate $0.1,0.5$ and $1.0 \%$ bile concentrations after $24 \mathrm{~h}$. Aflatoxin levels decreases significantly from $50 \mathrm{ppb}$ to $2.0 \mathrm{ppb}$ in control and to $1.8 \mathrm{ppb}$ in the ginger samples. High quantity of ginger reduced acceptability. This study demonstrated that ginger has no negative effects on LAB and aflatoxin level reduction potential of LAB, its use as additive in ogi could be encouraged.

Keywords: Ginger; Fermentation; Ogi; Aflatoxin; Lactic Acid Bacteria Load

\section{Introduction}

Ogi (maize pap), a fermented cereal porridge produced by lactic acid fermentation of maize (Zea mays), guinea corn (Sorghum bicolor) or millet
(Pennisetumtyphodenum), is a dietary staple food for children and adults in Nigeria often flavoured with ginger, garlic and onion [1]. It is produced by soaking corn in water for 2 to 3 days, followed by milling and sieving through a screen mesh. The sieved material is allowed to 


\section{Food Science and Nutrition Technology}

sediment and ferment, and the fermented product marketed as wet cakes locally known as ogi [2]. Different genera of microorganisms have been isolated during fermentation of maize into ogi, including various yeast and mould species, and Lactobacillus spp [3].

Lactic acid bacteria (LAB) are integral to many African fermented foods [4]. Today, LAB are a focus of intensive international research for their essential role in most fermented foods and ability to produce various antimicrobial compounds, thereby promoting probiotic properties [5].

Lactobacillus species are the predominant organisms involved in the fermentation of cereal based foods and beverages in Africa. These organisms are reported to have bacteriostatic, bactericidal, viricidal, anti-leukaemic and antitumor effects in the consumer [6]. Beneficial starter cultures are not usually used in the fermentation of traditional cereal based foods and beverages. However, it is reported that fermented foods have a probiotic potential [7] due to the probiotic Lactobacillus species that may be contained in them, some of which are of human intestinal origin [8].

Mycotoxins are natural secondary metabolites produced by fungi on agricultural commodities in field and during storage, under a wide range of climatic conditions and are of significance in food safety [9]. The food borne mycotoxins that are likely to be of great significance to human health in tropical developing countries are the fumonisins and aflatoxins [10]. Among these mycotoxins, aflatoxin has gained considerable attention because it is a more toxic and potent carcinogen even in small quantities [11] and it is also the mycotoxin of public health importance within the West African region.

Aflatoxins are toxic metabolites produced by a variety of moulds such as Aspergillus flavus and Aspergillus parasiticus. They are carcinogenic and can be present in grains, nuts, cottonseeds and other commodities associated with human food or animal feeds. Crops may be contaminated by one or more of the four following subtypes of aflatoxin: B 1, B 2, G 1 and G 2. Aflatoxin B 1 is the most toxic and frequently detected form. The other types present a significant danger at a high level concentration. Aflatoxins have been implicated in human health disorders including hepatocellular carcinoma, aflatoxicosis, Rey's syndrome and chronic hepatitis [12].

Accurate and rapid determination of the presence of aflatoxin in commodities is of paramount importance. The
Standards Organization of Nigeria (SON) sets standards on many food commodities, taking into account global standards as well as national production and target export markets. While it is generally recognized globally that there is no safe level of aflatoxin exposure, SON has set the maximum acceptable limit for maize grain at $4 \mathrm{ppb}$ for total aflatoxins and $2 \mathrm{ppb}$ for aflatoxin B1 [12].

Ginger (zingiber officinale roscoe, Zingiberacae) is an important medicinal plant which indigenous to several countries and is consumed worldwide as a spice and flavouring agent from the ancient time [13]. It has some tremendous beneficial effect to human body to cure various types of diseases due to its many chemical constituents such as Amaldehyde, Gingerol, Shogaol, and Paradol. Ginger is rich in antioxidants and phytochemicals with anti-inflammatory, antimicrobial and anticancer properties. It has been used as a condiment and for the treatment of ailments for many years.

However, there are limited studies on the antioxidant and scavenging power of ginger used to ferment ogi on aflatoxin levels and Lactic Acid Bacteria; one of the predominant fermenter and health benefiting organism in ogi. Since heavily aflatoxin-contaminated grain is a common feature in Nigerian markets, and ogi been food of many in Nigeria, this study therefore was aimed at determining the effect of ginger on lactic acid bacteria load in ogi, its aflatoxin reducing potential and other probiotic potentials when it is used as a co-fermenter of maize naturally infected with aflatoxin used for ogi production.

\section{Materials and Methods}

\section{Collection of Maize and Ginger Samples / Preparation}

Maize and Ginger for this study were obtained from Animal Care Consult, Ogere Remo, Abeokuta, Ogun State, Nigeria. The grains were sorted to remove dirts and spoilt ones from the healthy ones. Ginger was prepared according to modified methods of Olubamiwa AO [14]. Four hundred grams of maize were weighed into five bowls each and $1000 \mathrm{ml}$ of water added with ginger of 0 (no ginger), 4, 6, 8 and 10 grams incorporated respectively. Given rise to five different samples (A- $400 \mathrm{~g}$ maize + No ginger [control], $B-400 \mathrm{~g}$ maize $+4 \mathrm{~g}$ ginger. $C$ $-400 \mathrm{~g}$ maize $+6 \mathrm{~g}$ ginger, $\mathrm{D}-400 \mathrm{~g}$ maize $+8 \mathrm{~g}$ ginger and $\mathrm{E}-400 \mathrm{~g}$ maize $+10 \mathrm{~g}$ ginger). Each bowl of maize grains was fermented $24 \mathrm{~h}, 48 \mathrm{~h}, 72 \mathrm{~h}$ and $96 \mathrm{~h}$ respectively. For Aflatoxin, the zero hour samples were milled and analysed immediately and were used as control. 


\section{Food Science and Nutrition Technology}

\section{Acidity Changes}

Changes in the $\mathrm{pH}$ and TTA were assessed in triplicate at $12 \mathrm{~h}$ interval throughout the fermentation period. The $\mathrm{pH}$ was determined using a Metrohm $620 \mathrm{pH}$ meter (Metrohm Herisau, Switzerland) with a reference glass electrode. The $\mathrm{pH}$ meter was calibrated prior to each reading with standard buffer 7 . the TTA was analyzed by titrating $20 \mathrm{ml}$ of the supernatant against $0.1 \mathrm{~N} \mathrm{NaOH}$ until $\mathrm{pH} 8.30$ was attained. The titer volume of each homogenates was multiplied by 0.09 to give the percentage TTA as lactic acid [15].

\section{Acid Tolerance}

This was carried out by a modified method described by Haller, et al. (2001) [16]. The isolated lactobacilli were subjected to primary screening for acid tolerance in MRS broth adjusted to $\mathrm{pH} 2.5$ with $1 \mathrm{~N} \mathrm{HCl}$ for $90 \mathrm{~min}$ at $37^{\circ} \mathrm{C}$. The determination of survival was performed by single streaking on MRS agar plates, and the growth was observed after $24 \mathrm{~h}$ of anaerobic incubation at $37^{\circ} \mathrm{C}$. The growths of isolates on the agar were considered to be acid tolerant strains. These strains were selected and cultivated in MRS broth under anaerobic atmosphere at $37^{\circ} \mathrm{C}$.

Cultures $(106 \mathrm{cfu} / \mathrm{mL})$ were inoculated in $10 \mathrm{~mL}$ of $0.05 \mathrm{M}$ sodium phosphate buffer adjusted to $\mathrm{pH} 2.0$, 3.0, 4.0 and 7.0 with $1 \mathrm{~N} \mathrm{HCl}$. Samples were incubated at $37^{\circ} \mathrm{C}$ for $2 \mathrm{~h}$. Cultures were serially diluted using phosphate buffer $\mathrm{pH}$ 7.0. Appropriate dilution was plated on MRS agar for determination of viable cells after $24 \mathrm{~h}$ of incubation. The survival rate was calculated as the percentage of colonies grown on MRS agar compared to the initial cell concentration. Each experiment was performed in triplicate [16]. Initial counts were standardized using McFarland standard to $1.5 \times 106$ $\mathrm{cfu} / \mathrm{mL}$ before exposing isolates to variable bile concentrations.

\section{Bile Salt Tolerance}

To determine the effect of bile salts on the growth rate of LAB a modified method described by Liong and Shah (2005) [17] was employed. The MRS broths at concentrations of $0.5 \%, 1.0 \%$ and $1.5 \%(\mathrm{w} / \mathrm{v})$ of oxgall, Difco, Detroit, USA was prepared and dispensed in $10 \mathrm{~mL}$ volumes and sterilized by heating at $121^{\circ} \mathrm{C}$ for $15 \mathrm{~min}$. Pure cultures of each of the isolated LAB $(106 \mathrm{cfu} / \mathrm{mL})$ were inoculated into each medium, incubated at $37^{\circ} \mathrm{C}$ for 24 h., and growth monitored. The survival rate of each strain was expressed as the percentage of viable cells in the presence of bile salt compared to that without bile salt
(I.e, initial count before exposure to bile and final counts after exposure). The experiment was performed in triplicate and the mean values were calculated [17].

NOTE: Before exposure to different concentrations of bile, counts from each isolates were adjusted to $1.30 \mathrm{x}$ $106 \mathrm{cfu} / \mathrm{mL}$ using A $0.5 \mathrm{McF}$ arland standard.

Bile tolerance $=($ No. of viable cells with bile salts $) /$ (No. of viable cells without bile salts) $\times 100$

\section{Cholesterol-Lowering Property}

The ability of isolates to assimilate cholesterol was determined by a modified method described by Dora and Glenn (2002) [18].

A $9.9 \mathrm{ml}$ aliquot of MRS broth containing $0.4 \%$ bile salt $(\mathrm{w} / \mathrm{v})$ and $0.01 \%(\mathrm{w} / \mathrm{v})$ cholesterol was inoculated separately with $0.1 \mathrm{ml}$ overnight culture of each of the isolates. The inoculated bottles were then incubated at $37^{\circ} \mathrm{C}$ under anaerobic conditions for $18 \mathrm{~h}$. The bacterial cells were then removed from the culture broth by centrifugation at $4000 \mathrm{rpm}$ for $20 \mathrm{~min}$ and the supernatant was used directly for determination of cholesterol level. Spectrophotometer was used to determine the level of turbidity of each isolates supernatant. Less turbidity implies high reduction in cholesterol while high turbidity implies low reduction.

\section{Sensory Evaluation}

Sensory evaluation was done according to modified method described by Iwe, 2002 [19] using a ten member panel on a nine point Hedonic scale ranging from $9=$ high acceptability to $1=$ low acceptability.

\section{ELISA test for Total Aflatoxin Level}

The total aflatoxin level in was detected and quantified using Enzyme Linked Immunosorbent Assay (ELISA) kits (Agra Quant Aflatoxin. Singapore). The kit was used according to the manufacturer's instruction.

\section{Bacterial Genomic DNA Analysis}

Bacterial genomic DNA was extracted using the protocol stated by Trindade, et al. (2007) [20].

\section{Statistical Analysis}

Data obtained were inputted using SPSS (version 17.0). Significant differences between means $(p<0.05)$ were separated using Duncan multiple range test. 


\section{Food Science and Nutrition Technology}

\section{Results \& Discussion}

A significant decrease in $\mathrm{pH}$ value (Figure 1) throughout the fermentation period was noticed, while there was a significant increase (Figure 2) in the total titratable acidity (TTA) in all the samples. Steady decrease in $\mathrm{pH}$ and increase in TTA during fermentation of maize without ginger into ogi has been reported by Omemu, (2011) [3] this might be as a result of the consumption of free sugar in the sample and production of lactic acid by fermentative organisms responsible for the fermentation of ogi. In this study, the decrease in pH and TTA (Figures 1 and 2) was higher in the ginger samples compared to the non ginger samples.

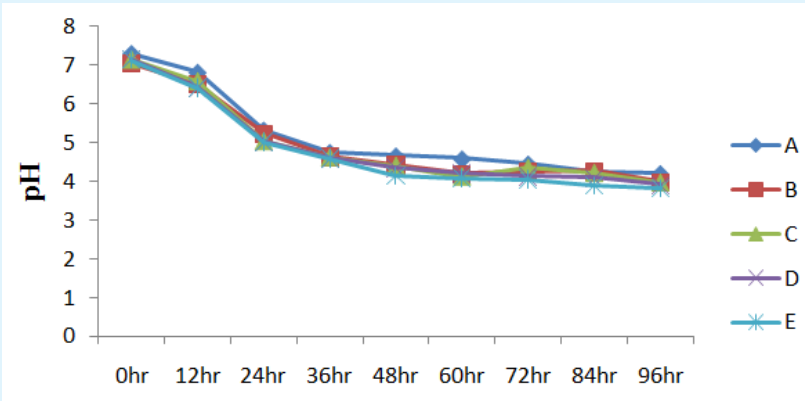

FERMENTATION TIME (HOURS)

Figure 1: Effect of fermentation on $\mathrm{pH}$ of ginger and non - ginger fermented maize. KEY: $A=$ Control ( 400 grams Maize No Ginger), $B=400$ grams maize +4 grams Ginger, $\mathrm{C}=400$ grams maize +6 grams Ginger, $D=400$ grams maize +8 grams Ginger, $E=400$ grams maize +10 grams Ginger.
Values are means of triplicate treatments.

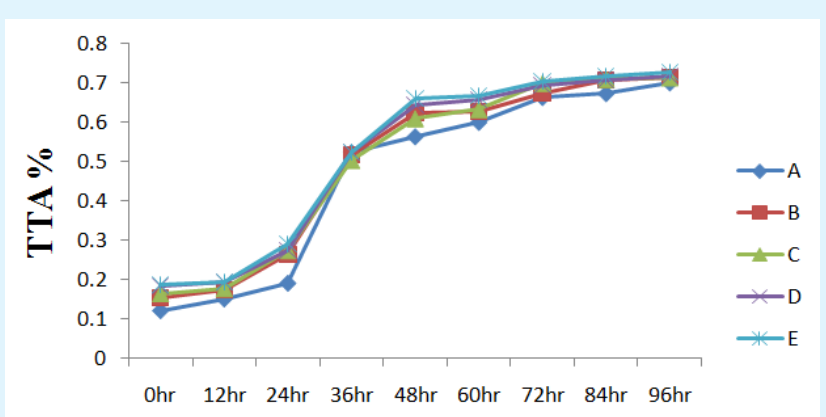

FERMENTATION TIME (HOURS)

Figure 2: Effect of fermentation on $\mathrm{pH}$ of ginger and non - ginger fermented maize. KEY: $A=$ Control $(400$ grams Maize No Ginger), $B=400$ grams maize +4 grams Ginger,, $\mathrm{C}=400$ grams maize +6 grams Ginger, $\mathrm{D}=400$ grams maize +8 grams Ginger, $\mathrm{E}=400$ grams maize +10 grams Ginger.

Values are means of triplicate treatments.

The antimicrobial activity of ginger has been reported [21]. But in the present study, ginger did not negatively affect LAB population as show over the $96 \mathrm{~h}$ fermentation period (Table 1). Increase in LAB growth during fermentation of maize without ginger has been reported [3], but in the present study, the ginger samples recorded more growth compared to the non ginger samples (Table 1 ), this might be as a result of the stimulating effects of ginger used on the growth of the LAB isolates resulting in the enhanced acid production [22].

\begin{tabular}{|c|c|c|c|c|c|}
\hline \multicolumn{7}{|c|}{ Time Interval (Hours) / LAB Counts (Cfu/MI) } \\
\hline Ogi/Ginger Samples & $\mathbf{0}$ & $\mathbf{2 4}$ & $\mathbf{4 8}$ & $\mathbf{7 2}$ & $\mathbf{9 6}$ \\
\hline A & 0 & $3.0 \pm 0.033^{\mathrm{a}}$ & $5.8 \pm 0.033^{\mathrm{b}}$ & $7.6 \pm 0.033^{\mathrm{cd}}$ & $8.2 \pm 0.067^{\mathrm{d}}$ \\
\hline B & 0 & $3.3 \pm 1.333^{\mathrm{ab}}$ & $5.9 \pm 0.33^{\mathrm{b}}$ & $7.7 \pm 0.333^{\mathrm{c}}$ & $8.5 \pm 1.454^{\mathrm{d}}$ \\
\hline C & 0 & $3.4 \pm 2.000^{\mathrm{ab}}$ & $5.8 \pm 0.66^{\mathrm{b}}$ & $7.7 \pm 0.333^{\mathrm{cd}}$ & $8.8 \pm 0.882^{\mathrm{d}}$ \\
\hline D & 0 & $3.4 \pm 1.453^{\mathrm{ab}}$ & $5.9 \pm 0.333^{\mathrm{b}}$ & $7.7 \pm 0.578^{\mathrm{c}}$ & $8.8 \pm 0.882^{\mathrm{d}}$ \\
\hline E & 0 & $3.5 \pm 1.202^{\mathrm{ab}}$ & $6.0 \pm 0.882^{\mathrm{b}}$ & $7.8 \pm 0.577^{\mathrm{c}}$ & $8.7 \pm 1.202^{\mathrm{d}}$ \\
\hline
\end{tabular}

Table 1: Lactic Acid Bacteria Count of Fermenting Maize Containing Different Quantities of Ginger.

Values are means of triplicate treatments \pm standard error.

KEY: $A=$ control (400 grams maize no ginger), $B=400$ grams maize +4 grams ginger, $C=400$ grams maize +6 grams ginger, $D=400$ grams maize +8 grams ginger, $E=400$ grams maize +10 grams ginger. 


\section{Food Science and Nutrition Technology}

A decrease in total aflatoxin level of naturally aflatoxin contaminated maize was also studied and a decrease in total aflatoxin level was recorded among all the samples (Table 2). Reduction in aflatoxin level in fermented naturally aflatoxin contaminated maize without ginger has been reported by Oluwafemi and Ikeowo, but in the present study, a more decrease was noticed among the ginger samples compared to the non ginger sample.

\begin{tabular}{|c|c|c|c|c|}
\hline \multirow{2}{*}{ Time (hrs) } & \multicolumn{5}{|c|}{ Total Aflatoxin level (ppb) at different } \\
& quantities of Ginger \\
\hline & $\mathrm{A}$ & $\mathrm{B}$ & $\mathrm{C}$ & $\mathrm{D}$ \\
\hline 0 & 50 & 50 & 50 & 50 \\
\hline 24 & $4.0 \pm 0.23$ & $4.0 \pm 0.23$ & $4.0 \pm 0.23$ & $4.0 \pm 0.23$ \\
\hline 48 & $3.2 \pm 0.17$ & $3.0 \pm 0.17$ & $3.0 \pm 0.23$ & $3.0 \pm 0.15$ \\
\hline 72 & $2.0 \pm 0.17$ & $1.9 \pm 0.17$ & $2.0 \pm 015$ & $1.9 \pm 0.23$ \\
\hline 96 & $2.0 \pm 0.17$ & $1.9 \pm 0.23$ & $1.9 \pm 0.17$ & $1.8 \pm 0.16$ \\
\hline
\end{tabular}

Table 2: Aflatoxin levels of fermenting maize containing different quantities of ginger.

Values are means of triplicate treatments \pm standard error.

The use of ginger as additive in ogi has lots of benefits ranging from health [23] to improvement of ogi shelf life, but at high concentrations in ogi, people tends to dislike it due to its peppery taste (Figure 3 ) as shown in this study.

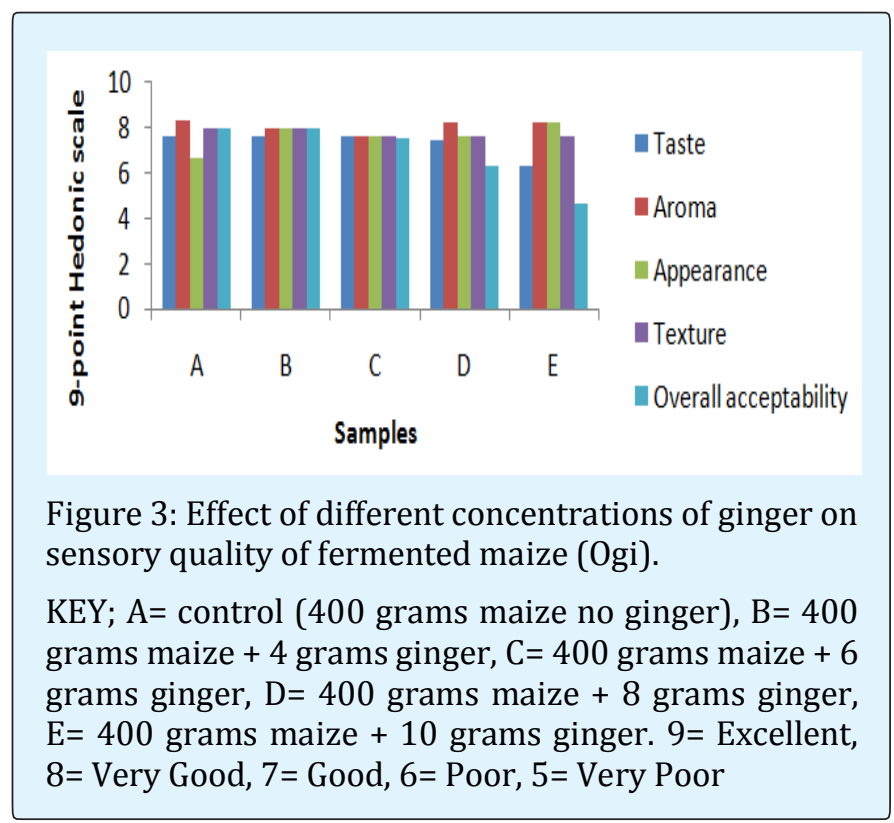

Survival of bacterial strains in low $\mathrm{pH}$ and Bile salt is a more accurate indication of the ability of strains to survive passage through the stomach. Result (Tables 3 and 4) shows that isolates could survive $\mathrm{pH}$ and bile concentrations after $24 \mathrm{~h}$ exposure to $\mathrm{pH}$ of 7, 2, 3 and 4 as well as $0.1,0.51 \%$ bile, although a decrease in growth was noticed among all the samples. Overall, the ginger samples recorded more growth compared to the non ginger samples.

\begin{tabular}{|c|c|c|c|c|c|c|c|c|c|c|c|c|c|c|c|c|c|c|c|c|}
\hline \multirow{3}{*}{ Isolates } & \multicolumn{20}{|c|}{ Bacterial count $\left(\times 10^{6} \mathrm{CFU} / \mathrm{mL}\right)$ after $24 \mathrm{hrs}$ of acid exposure } \\
\hline & \multicolumn{5}{|c|}{ pH7 } & \multicolumn{5}{|c|}{ pH4 } & \multicolumn{5}{|c|}{ pH3 } & \multicolumn{5}{|c|}{ pH2 } \\
\hline & A & $\mathrm{B}$ & $\mathrm{C}$ & $\mathrm{D}$ & E & A & B & $\mathrm{C}$ & D & E & A & B & $\mathrm{C}$ & D & E & A & B & $\mathrm{C}$ & D & E \\
\hline L. plantarum & $\begin{array}{l}1.53 \pm \\
0.03^{\mathrm{b}}\end{array}$ & $=\begin{array}{l}1.83 \pm \\
0.04^{b}\end{array}$ & $\begin{array}{c}1.71 \pm \\
.03^{a}\end{array}$ & $\begin{array}{l}1.73 \pm \\
0.02^{b}\end{array}$ & $\begin{array}{l}1.83 \pm \\
0.02^{b}\end{array}$ & $\begin{array}{c}1.46 \pm \\
0.03^{b}\end{array}$ & $\begin{array}{l}1.80 \pm \\
0.03^{\mathrm{ab}}\end{array}$ & $\begin{array}{l}1.59 \pm \\
0.02^{\mathrm{ab}}\end{array}$ & $\begin{array}{l}1.61 \pm \\
0.02^{b}\end{array}$ & $\begin{array}{l}1.75 \pm \\
0.03^{b}\end{array}$ & $\begin{array}{l}1.24 \pm \\
0.02^{\mathrm{a}}\end{array}$ & $\begin{array}{l}1.74 \pm \\
0.03^{\mathrm{ab}}\end{array}$ & $\begin{array}{l}1.48 \pm \\
0.03^{\mathrm{bc}}\end{array}$ & $\begin{array}{l}1.43 \pm \\
0.03^{b}\end{array}$ & $\begin{array}{l}1.60 \pm \\
0.04^{b}\end{array}$ & $\begin{array}{c}1.22 \pm \\
0.03^{\mathrm{c}}\end{array}$ & $\begin{array}{l}1.63 \pm \\
0.09^{\mathrm{a}}\end{array}$ & $=\begin{array}{l}1.40 \pm \\
0.03^{c}\end{array}$ & $\begin{array}{l}1.40 \pm \\
0.03^{\mathrm{c}}\end{array}$ & $\begin{array}{l}1.37 \pm \\
0.04^{\mathrm{a}}\end{array}$ \\
\hline L. fermentum & $\begin{array}{l}1.70 \pm \\
0.03^{c}\end{array}$ & $=\begin{array}{l}1.71 \pm \\
0.03^{\mathrm{a}}\end{array}$ & $\begin{array}{l}1.70 \pm \\
0.03^{c}\end{array}$ & $\begin{array}{l}1.80 \pm \\
0.03^{b}\end{array}$ & $\begin{array}{l}1.84 \pm \\
0.03^{\mathrm{b}}\end{array}$ & $\begin{array}{l}1.63 \pm \\
0.02^{\mathrm{bc}}\end{array}$ & $\begin{array}{l}1.51 \pm \\
0.02^{\mathrm{ab}}\end{array}$ & $\begin{array}{l}1.63 \pm \\
0.02^{b}\end{array}$ & $\mid \begin{array}{l}1.71 \pm \\
0.02^{c}\end{array}$ & $\begin{array}{l}1.80 \pm \\
0.02^{\mathrm{b}}\end{array}$ & $\begin{array}{l}1.60 \pm \\
.03^{\mathrm{b}}\end{array}$ & $\begin{array}{l}1.48 \pm \\
0.03^{\mathrm{bc}}\end{array}$ & $\begin{array}{l}1.60 \pm \\
0.03^{\mathrm{b}}\end{array}$ & \begin{tabular}{|l}
$1.70 \pm$ \\
$0.02^{\mathrm{bc}}$
\end{tabular} & $\begin{array}{l}1.75 \pm \\
0.01^{\mathrm{b}}\end{array}$ & $\begin{array}{l}1.35 \pm \\
0.03^{\mathrm{a}}\end{array}$ & $\begin{array}{l}1.40 \pm \\
0.03^{\mathrm{c}}\end{array}$ & $\mid \begin{array}{l}1.35 \pm \\
0.03^{\mathrm{a}}\end{array}$ & $\begin{array}{l}1.60 \pm \\
0.02^{\mathrm{a}}\end{array}$ & $\begin{array}{c}1.65 \pm \\
0.03^{\mathrm{a}}\end{array}$ \\
\hline L. acidophilus & $\begin{array}{l}1.73 \pm \\
0.03^{\mathrm{a}}\end{array}$ & $=\begin{array}{l}1.40 \pm \\
0.03^{\mathrm{b}}\end{array}$ & $\begin{array}{l}1.62 \pm \\
0.03^{a}\end{array}$ & $\begin{array}{l}1.67 \pm \\
0.04^{\mathrm{ab}}\end{array}$ & $\begin{array}{l}1.87 \pm \\
0.04^{c}\end{array}$ & $\begin{array}{l}1.60 \pm \\
0.03^{\mathrm{a}}\end{array}$ & $\begin{array}{l}1.34 \pm \\
0.03^{c}\end{array}$ & $\begin{array}{l}1.53 \pm \\
0.03^{b}\end{array}$ & $\mid \begin{array}{l}1.60 \pm \\
0.02^{\mathrm{a}}\end{array}$ & $\begin{array}{l}1.80 \pm \\
0.02^{c}\end{array}$ & $\begin{array}{l}1.55 \pm \\
0.03^{\mathrm{a}}\end{array}$ & $\begin{array}{l}1.30 \pm \\
0.04 \mathrm{~b}\end{array}$ & $\begin{array}{l}1.52 \pm \\
0.03^{\mathrm{a}}\end{array}$ & $\begin{array}{c}1.60 \pm \\
0.02^{\mathrm{a}}\end{array}$ & $\begin{array}{l}1.37 \pm \\
0.04^{b}\end{array}$ & $\begin{array}{l}1.53 \pm \\
0.03^{a}\end{array}$ & $\begin{array}{l}1.27 \pm \\
0.02^{\mathrm{a}}\end{array}$ & $=\begin{array}{l}1.44 \pm \\
0.03^{\mathrm{b}}\end{array}$ & $\begin{array}{l}1.50 \pm \\
0.02^{b}\end{array}$ & $\begin{array}{l}1.23 \pm \\
0.02^{\mathrm{a}}\end{array}$ \\
\hline L. brevis & $\begin{array}{l}1.43 \pm \\
0.02^{\mathrm{a}}\end{array}$ & $=\begin{array}{l}1.48 \pm \\
0.02^{\mathrm{b}}\end{array}$ & $\begin{array}{l}1.51 \pm \\
0.02^{c}\end{array}$ & $\begin{array}{l}1.55 \pm \\
0.02^{c d}\end{array}$ & $\begin{array}{l}1.67 \pm \\
0.02^{\mathrm{d}}\end{array}$ & $\begin{array}{l}1.37 \pm \\
0.04^{\mathrm{a}}\end{array}$ & $\begin{array}{l}1.42 \pm \\
0.02^{\mathrm{b}}\end{array}$ & $\begin{array}{l}1.43 \pm \\
0.02^{\mathrm{b}}\end{array}$ & $\begin{array}{l}1.45 \pm \\
0.03^{c}\end{array}$ & $\begin{array}{l}1.50 \pm \\
0.03^{\mathrm{c}}\end{array}$ & $\begin{array}{l}1.31 \pm \\
0.01^{\mathrm{b}}\end{array}$ & $\begin{array}{l}1.31 \pm \\
0.02^{\mathrm{a}}\end{array}$ & $\begin{array}{l}1.33 \pm \\
0.02^{\mathrm{a}}\end{array}$ & $\begin{array}{l}1.35 \pm \\
0.04^{b}\end{array}$ & $\begin{array}{l}1.37 \pm \\
0.04^{\mathrm{b}}\end{array}$ & $\begin{array}{l}1.23 \pm \\
0.05^{c}\end{array}$ & $\begin{array}{l}1.20 \pm \\
0.02^{\mathrm{a}}\end{array}$ & $=\begin{array}{l}1.22 \pm \\
0.02^{\mathrm{b}}\end{array}$ & $\begin{array}{l}1.22 \pm \\
0.02^{\mathrm{b}}\end{array}$ & $\begin{array}{l}1.25 \pm \\
0.02^{\mathrm{c}}\end{array}$ \\
\hline
\end{tabular}

Table 3: Effect of $\mathrm{pH}$ on the viability of the isolated LAB strains

Values are means of triplicate treatment \pm standard error.

( $F=46.823, P=0.001$, superscript $a, b, c, d$ are significantly different $(p<0.05)$.

KEY: $A=$ Control (400 grams Maize No Ginger), $B=400$ grams maize +4 grams Ginger,,$C=400$ grams maize +6 grams

Ginger, $D=400$ grams maize +8 grams Ginger, $E=400$ grams maize +10 grams Ginger 


\section{Food Science and Nutrition Technology}

\begin{tabular}{|c|c|c|c|c|c|c|c|c|c|c|c|c|c|c|c|}
\hline \multirow{3}{*}{ Isolates } & \multicolumn{15}{|c|}{ Bacterial count $\left(\times 10^{6} / \mathrm{CFUmL}\right)$ after 24 hrs of exposure to different bile concentrations } \\
\hline & \multicolumn{5}{|c|}{ Bile 0.1\% } & \multicolumn{5}{|c|}{ Bile 0.5\% } & \multicolumn{5}{|c|}{ Bile 1\% } \\
\hline & $\mathrm{A}$ & $\mathrm{B}$ & $\mathrm{C}$ & $\mathrm{D}$ & E & $\mathrm{A}$ & $\mathrm{B}$ & $\mathrm{C}$ & $\mathrm{D}$ & E & A & $\mathrm{B}$ & $\mathrm{C}$ & $\mathrm{D}$ & E \\
\hline $\begin{array}{c}L . \\
\text { plantarum }\end{array}$ & $\begin{array}{l}1.61 \pm \\
0.02^{b}\end{array}$ & $\begin{array}{l}1.58 \pm \\
0.04^{\mathrm{b}}\end{array}$ & $\begin{array}{l}1.53 \pm \\
0.02^{\mathrm{b}}\end{array}$ & $\begin{array}{l}1.80 \pm \\
0.02^{\mathrm{c}}\end{array}$ & $\begin{array}{l}1.43 \pm \\
0.02^{\mathrm{a}}\end{array}$ & $\begin{array}{l}1.53 \pm \\
0.03^{\mathrm{ab}}\end{array}$ & $\begin{array}{l}1.40 \pm \\
0.03^{\mathrm{b}}\end{array}$ & $\begin{array}{l}1.43 \pm \\
0.03^{\mathrm{a}}\end{array}$ & $\begin{array}{l}1.71 \pm \\
0.01^{b}\end{array}$ & $\begin{array}{l}1.40 \pm \\
0.03^{\mathrm{a}}\end{array}$ & $\begin{array}{l}1.50 \pm \\
0.03^{\mathrm{a}}\end{array}$ & $\begin{array}{l}1.37 \pm \\
0.03^{\mathrm{a}}\end{array}$ & $\begin{array}{l}1.37 \pm \\
0.04^{\mathrm{a}}\end{array}$ & $\begin{array}{l}1.51 \pm \\
0.03^{\mathrm{a}}\end{array}$ & $\begin{array}{l}1.37 \pm \\
0.03^{\mathrm{a}}\end{array}$ \\
\hline L. fermentum & $\begin{array}{l}1.73 \pm \\
0.03^{b}\end{array}$ & $\begin{array}{l}1.51 \pm \\
0.02^{\mathrm{b}}\end{array}$ & $\begin{array}{l}1.71 \pm \\
0.03^{\mathrm{c}}\end{array}$ & $\begin{array}{l}1,48 \pm \\
0.02^{b}\end{array}$ & $\begin{array}{l}1.23 \pm \\
0.03^{b}\end{array}$ & $\begin{array}{l}1.43 \pm \\
0.03^{\mathrm{a}}\end{array}$ & $\begin{array}{l}1.43 \pm \\
0.02^{\mathrm{a}}\end{array}$ & $\begin{array}{l}1.62 \pm \\
0.00^{\mathrm{b}}\end{array}$ & $\begin{array}{l}1.44 \pm \\
0.03^{\mathrm{b}}\end{array}$ & $\begin{array}{l}1.11 \pm \\
0.02^{\mathrm{a}}\end{array}$ & $\begin{array}{l}1.40 \pm \\
0.03^{\mathrm{a}}\end{array}$ & $\begin{array}{l}1.37 \pm \\
0.02^{\mathrm{a}}\end{array}$ & $\begin{array}{l}1.53 \pm \\
0.03^{\mathrm{a}}\end{array}$ & $\begin{array}{l}1.43 \pm \\
0.02^{\mathrm{b}}\end{array}$ & $\begin{array}{l}1.38 \pm \\
0.02^{c}\end{array}$ \\
\hline L. acidophilus & $\begin{array}{l}1.67 \pm \\
0.03^{b}\end{array}$ & $\begin{array}{l}1.39 \pm \\
0.03^{\mathrm{c}}\end{array}$ & $\begin{array}{l}1.67 \pm \\
0.03^{b}\end{array}$ & $\begin{array}{l}1.43 \pm \\
0.02^{b}\end{array}$ & $\begin{array}{l}1.23 \pm \\
0.03^{a}\end{array}$ & $\begin{array}{l}1.55 \pm \\
0.03^{\mathrm{a}}\end{array}$ & $\begin{array}{l}1.31 \pm \\
0.03^{b}\end{array}$ & $\begin{array}{l}1.59 \pm \\
0.03^{\mathrm{a}}\end{array}$ & $\begin{array}{l}1.40 \pm \\
0.03^{b}\end{array}$ & $\begin{array}{l}1.27 \pm \\
0.04^{\mathrm{ab}}\end{array}$ & $\begin{array}{l}1.51 \pm \\
0.02^{\mathrm{a}}\end{array}$ & $\begin{array}{l}1.39 \pm \\
0.03^{\mathrm{c}}\end{array}$ & $\begin{array}{l}1.56 \pm \\
0.02^{\mathrm{a}}\end{array}$ & $\begin{array}{l}1.32 \pm \\
0.02^{\mathrm{a}}\end{array}$ & $\begin{array}{l}1.35 \pm \\
0.03^{b}\end{array}$ \\
\hline L. brevis & $\begin{array}{l}1.37 \pm \\
0.02^{\mathrm{c}}\end{array}$ & $\begin{array}{l}1.37 \pm \\
0.02^{\mathrm{c}}\end{array}$ & $\begin{array}{l}1.34 \pm \\
0.02^{\mathrm{a}}\end{array}$ & $\begin{array}{l}1.25 \pm \\
0.02^{\mathrm{a}}\end{array}$ & $\begin{array}{l}1.25 \pm \\
0.02^{\mathrm{a}}\end{array}$ & $\begin{array}{l}1.32 \pm \\
0.02^{\mathrm{b}}\end{array}$ & $\begin{array}{l}1.32 \pm \\
0.02^{\mathrm{b}}\end{array}$ & $\begin{array}{l}1.41 \pm \\
0.03^{\mathrm{c}}\end{array}$ & $\begin{array}{l}1.41 \pm \\
0.03^{\mathrm{c}}\end{array}$ & $\begin{array}{l}1.41 \pm \\
0.03^{\mathrm{c}}\end{array}$ & $\begin{array}{l}1.12 \pm \\
0.02^{\mathrm{ab}}\end{array}$ & $\begin{array}{l}1.34 \pm \\
0.03^{\mathrm{b}}\end{array}$ & $\begin{array}{l}1.34 \pm \\
0.03^{\mathrm{b}}\end{array}$ & $\begin{array}{l}1.34 \pm \\
0.03^{\mathrm{b}}\end{array}$ & $\begin{array}{l}1.30 \pm \\
0.03^{\mathrm{a}}\end{array}$ \\
\hline
\end{tabular}

Table 4: Bile Tolerance of LAB (Cfu/mL) at Different Bile Concentrations.

Values are means of triplicate treatment \pm standard error.

$\mathrm{F}=35.823, \mathrm{P}=0.001$, superscript $\mathrm{a}, \mathrm{b}, \mathrm{c}, \mathrm{d}$ are significantly different $(\mathrm{P}<0.05)$.

KEY: $A=$ Control (400 grams Maize No Ginger), $B=400$ grams maize +4 grams Ginger,,$C=400$ grams maize +6 grams Ginger, $D=400$ grams maize +8 grams Ginger, $E=400$ grams maize +10 grams Ginger.

This might be as a result of the stimulating effects of ginger used on the growth of the LAB isolates resulting in the enhanced acid production [22].

These tolerances are an indication that the isolates could successfully transit the human stomach and may be capable of reaching the intestinal environment to carry out their functioning effects.

Another phenomenon related to the presence of the deconjugation activity is the reduction of serum cholesterol. The removal of cholesterol by lactobacilli in vitro could be due to an uptake or assimilation of cholesterol by bacterial strains. Liong MT, et al. demonstrated that a portion of the cholesterol assimilated by Lactobacillus strains was incorporated into the cellular membrane [17]. Hyeong-Jun L, et al. Reported that strains of lactobacilli tested removed 31.5 to $58.5 \%$ cholesterol in the growth medium. In this study, L. plantarum shows the highest level $(41.23 \%)$ of cholesterol reduction (Tables 5 and 6) but the percentage of cholesterol removal varied considerably among strains. L. brevis showed no reduction or assimilation of cholesterol at the course of this study $[24,25]$.

\begin{tabular}{|c|c|c|c|c|c|}
\hline \multirow{2}{*}{ Isolates } & \multicolumn{5}{|c|}{ Percentage (\%) absorbance } \\
\cline { 2 - 6 } & $\mathrm{A}$ & $\mathrm{B}$ & $\mathrm{C}$ & $\mathrm{D}$ & $\mathrm{E}$ \\
\hline L.plantarum & 15.25 & 18.93 & 14.69 & 41.23 & 12.71 \\
\hline L. fermentum & 11.3 & 11.3 & 13.28 & 20.9 & 13.28 \\
\hline L.acidophilus & 17.51 & 12.15 & 17.51 & 20.9 & 20.9 \\
\hline L. brevis & - & - & - & - & - \\
\hline
\end{tabular}

Table 5: Cholesterol Assimilation of Isolated LAB on Fermented Maize.

KEY - = REDUCTION += NO REDUCTION.A= Control (400 grams Maize No Ginger), $B=400$ grams maize +4 grams Ginger, $\mathrm{C}=400$ grams maize +6 grams Ginger, $\mathrm{D}=400$ grams maize +8 grams Ginger, $\mathrm{E}=400$ grams maize +10 grams Ginger.

\begin{tabular}{|c|c|c|c|c|}
\hline Isolate code & $\begin{array}{c}\text { Description of closest } \\
\text { identity }\end{array}$ & E- Value & \% Identity & $\begin{array}{c}\text { Accession number of similarity } \\
\text { sequences }\end{array}$ \\
\hline 1 & $\begin{array}{c}\text { Lactobacillus } \\
\text { plantarumMF1298 }\end{array}$ & 0 & $99 \%$ & CP013149.1 \\
\hline 2 & Lactobacillus fermentum & 0 & $99 \%$ & FJ462686.1 \\
\hline 3 & $\begin{array}{c}\text { Lactobacillus acidophilus } \\
\text { EMBS082 }\end{array}$ & 0 & $99 \%$ & KC150145.1 \\
\hline
\end{tabular}

Table 6: The similarity of bacterial sequences with sequences obtained from NCBI database gene bank. 


\section{Food Science and Nutrition Technology}

\section{Conclusion}

Ginger does not have adverse effect on Lactobacilli isolated from ogi fermented with ginger. Hence, the use of ginger in ogi would decrease the chances of food poisoning, reduce the risks of food contamination, protect consumers from aflatoxin contamination and improve health status.

The use of ginger as food additive should be encouraged since it does not have a negative effect on $\mathrm{LAB}$ growth.

It was observed that, the Lactobacillus strains associated with ogi fermented with ginger also contain potential probiotic strains with cholesterol-lowering properties.

Therefore, the use of ginger in ogi and probably other food items would decrease the chances of food poisoning, reduce the risk of food contamination, protect the consumer from different food-borne diseases and improve health status by using a small quantity of it. It is therefore concluded that ginger do not reduce but enhance the growth LAB and subsequently lead to aflatoxin level reduction.

\section{References}

1. Ijabadeniyi AO (2007) microorganisms associated with ogi traditionally produced from three varieties of maize. Research Journal of Microbiology 2(3): 247253.

2. Akinrele IA (1968) Fermentation studies on maize during the preparation of a traditional African starch cake food. Journal of Science and Food Agriculture 21(12): 619-625.

3. Omemu AM (2011) Fermentation dynamics during production of ogi, a Nigerian fermented cereal porridge. Report and Opinion 3(4): 8-17.

4. Oluwajoba SO, Akinyosoye FA, Oyetayo VO (2013) In Vitro screening and selection of probiotic lactic acid bacteria isolated from spontaneously fermenting Kunu-zaki. Advances in Microbiology 3: 309-316.

5. Temmerman R, Pot B, Huys G, Swings J (2002) Identification and Antibiotic Susceptibility of Bacterial Isolates from Probiotic Products. International Journal of Food Microbiology 81: 1-10.
6. Iwuoha CI, Eke OS (1996) Nigerian indigenous fermented foods and their traditional process of operation, inherent problems, improvements and current status. Food Research International 29(5-6): 527-540.

7. Mugula JK, Narvhus JA, Sorhaug T (2003) Use of starter cultures of lactic acid bacteria and yeasts in the preparation of Togwa, a Tanzanian fermented food. International Journal of Food Microbiology 83 (3): 307-318.

8. Blandino A, Al-Aseeri ME, Pandiella SS, Cantero D, Webb C (2003) Cereal-Based Fermented Foods and Beverages. Food Research International 36(6): 527543.

9. Mady AI (2001) Deterioration and spoilage of peanuts and desiccated coconuts from two sub - Saharan tropical East African countries due to the associated mycobiota and other degradative enzymes. Mycopathologia 150(2): 67-84.

10. Committee on Toxicity of Chemicals in Food, Consumer Products and the Environment, COT (1997). A Statement on Ochratoxin A in dried wire fruit. Department of Health, London 143.

11. FAO (2000) United Nations Food and Agricultural Organization, Evaluation of Certain Mycotoxins in Food: Fifty-Sixth Report of the Joint FAO/WHO Expert Committee on Food Additives. World Health Organization, Geneva.

12. Sule EI, Orukotan A, Lado A, Adewumi AAJ (2015) Total aflatoxin level and fungi contamination of maize and maize products. African Journal of Food Science and Technology 6(8): 229-233.

13. Ghosh SA, Banerjee KH, Mullick I, Banerjee J (2011) Zingiber officinale: a natural gold. International Journal of Pharma and Bio Sciences 2(1): 283-294.

14. Ziaur-Rehman A, Salariya AM, Farzana H (2003) Antioxidant activity of ginger in sunflower oil. Journal of Food and Agricultural Science 83(7): 624-629.

15. Olubamiwa AO, Kolapo AL (2008) Evaluation of nutritional composition and acceptability of soycoconut milk-based yoghurt fermented with different starter culture. Food 2(1): 65-69.

16. Haller D, Colbus H, Ganzle MG, Scherenbacher P, Bode C, et al. (2001) Metabolic and Functional Properties of Lactic Acid Bacteria in the Gastrointestinal 


\section{Food Science and Nutrition Technology}

Ecosystem: A Comparative In Vitro Study between Bacteria of Intestinal and Fermented Food Origin. Systematic and Applied Microbiology 24: 218-226.

17. Liong MT, Shah NP (2005) Acid and bile tolerance and cholesterol removal ability of lactobacilli strains. Journal of Dairy Science 88: 55-66.

18. Dora IAP, Glenn RG (2002) Cholesterol Assimilation by Lactic Acid Bacteria and Bifidobacteria Isolated from the Human Gut. Applied Environmental Microbiology 68(9): 4689-4693.

19. Iwe MO (2002) Sensory Methods and Analysis. Uwani-Enugu: Rojoint communication Services 3467.

20. Trindade LC, Marques E, Lopes DB, Ferreira MASV (2007) Development of a molecular method for detection and identification of Xanthomonascampestrispv. viticola. Summa Phytopathologica 33(1): 16-23.

21. Kolapo AL, Popoola TOS, Sanni MO, Afolabi RO (2007) Peservation of soybean dadawa condiment with dichloromethane extract of ginger. Research Journal of Microbiology 3: 254-259.

22. Tiwari KP, Pandey A (1981) Effect of some essential oils on lactic acid bacteria. Journal of Food Science Research 3(81): 161-163.

23. Mahmoodi M, Islami MR, Karam AGR, Khaksari M, Sahebghadam LA, et al. (2006) Study of the effects of raw garlic consumption on the level of lipids and other blood biochemical factors in hyperlipidemic individuals. Pakistan Journal of Pharmaceutical Science 19(4): 295-298.

24. Hyeong-Jun L, So-Young K, Wan-Kyu L (2004) Isolation of cholesterol-lowering lactic acid bacteria from human intestine for probiotic use. Journal of veterinary science 5(4): 391-395.

25. Park DL, Pohland AE (1986) Arationale for the control of aflatoxin in Feeds. In Steyin PS, Vlegger M (Eds.), Elsevier, New York 473. 